\title{
One-Way Quantum Repeater Based on Near-Deterministic Photon-Emitter Interfaces
}

\author{
Johannes Borregaard $\odot,{ }^{1,2}$ Hannes Pichler, ${ }^{3,4}$ Tim Schröder, ${ }^{5,6}$ Mikhail D. Lukin, ${ }^{4}$ Peter Lodahl, ${ }^{6}$ and Anders S. Sørensen ${ }^{6}$ \\ ${ }^{1}$ QMATH, Department of Mathematical Sciences, University of Copenhagen, \\ DK-2100 Copenhagen $\varnothing$, Denmark \\ ${ }^{2}$ QuTech and Kavli Institute of Nanoscience, Delft University of Technology, \\ Lorentzweg 1, 2628 CJ Delft, The Netherlands \\ ${ }^{3}$ ITAMP, Harvard-Smithsonian Center for Astrophysics, Cambridge, Massachusetts 02138, USA \\ ${ }^{4}$ Department of Physics, Harvard University, Cambridge, Massachusetts 02138, USA \\ ${ }^{5}$ Department of Physics, Humboldt-Universität, 12489 Berlin, Germany \\ ${ }^{6}$ Center for Hybrid Quantum Networks $(H y-Q)$, The Niels Bohr Institute, University of Copenhagen, \\ DK-2100 Copenhagen $\varnothing$, Denmark
}

(Received 12 July 2019; revised manuscript received 15 April 2020; accepted 1 May 2020; published 30 June 2020)

\begin{abstract}
We propose a novel one-way quantum repeater architecture based on photonic tree-cluster states. Encoding a qubit in a photonic tree cluster protects the information from transmission loss and enables long-range quantum communication through a chain of repeater stations. As opposed to conventional approaches that are limited by the two-way communication time, the overall transmission rate of the current quantum repeater protocol is determined by the local processing time enabling very high communication rates. We further show that such a repeater can be constructed with as little as two stationary qubits and one quantum emitter per repeater station, which significantly increases the experimental feasibility. We discuss potential implementations with diamond defect centers and semiconductor quantum dots efficiently coupled to photonic nanostructures and outline how such systems may be integrated into repeater stations.
\end{abstract}

DOI: 10.1103/PhysRevX.10.021071

\section{INTRODUCTION}

Encoding information in quantum systems is the fundamental principle of quantum information technologies, ranging from quantum computers [1] to unconditionally secure communication [2]. Quantum networks constitute an important element for implementing such technologies in a scalable fashion [3]. The exact requirements and applications of large-scale quantum networks constitute an active research area [4]. One of the key challenges for constructing large-scale quantum networks is to faithfully transmit quantum information over long distances, which is challenging due to transmission loss.

Quantum repeaters have been proposed as a means to overcome transmission loss by exploiting quantum correlations to extend the transmission length of quantum information [5-7]. The conventional quantum repeater architecture relies on heralded quantum entanglement distribution, which necessitates long-lived quantum memories and two-way communication between sender and

Published by the American Physical Society under the terms of the Creative Commons Attribution 4.0 International license. Further distribution of this work must maintain attribution to the author(s) and the published article's title, journal citation, and DOI.
Subject Areas: Quantum Physics,

Quantum Information receiver [6]. The need for heralding limits the communication rate at which quantum information can be distributed and requires long-lived quantum memories with efficient light-matter coupling $[8,9]$. To overcome these limitations, one-way and all-photonic quantum repeaters have been proposed [10-16]. One-way repeaters use multiphoton encoding and quantum error-correcting codes to protect the quantum information from both loss and operational errors. In this way, quantum information can be transmitted from one repeater station to the next without the need for preestablished entangled links. For these reasons, in principle, one-way repeaters can significantly boost the distribution rate [7] without the need for a long-lived quantum memory for key applications such as long-distance quantum key distribution [17]. An outstanding challenge involving the physical implementation of one-way quantum repeaters is how to efficiently generate the multiqubit error-correcting codes and how to perform error correction. This usually requires many high-fidelity two-qubit operations and considerable amounts of auxiliary qubits at each repeater station $[12,15,16,18]$.

In this article, we propose a novel one-way quantum repeater architecture that can be implemented with as little as two memory qubits and one quantum emitter per repeater station. Our approach is based on photonic treecluster states [19], which are used to encode a message 


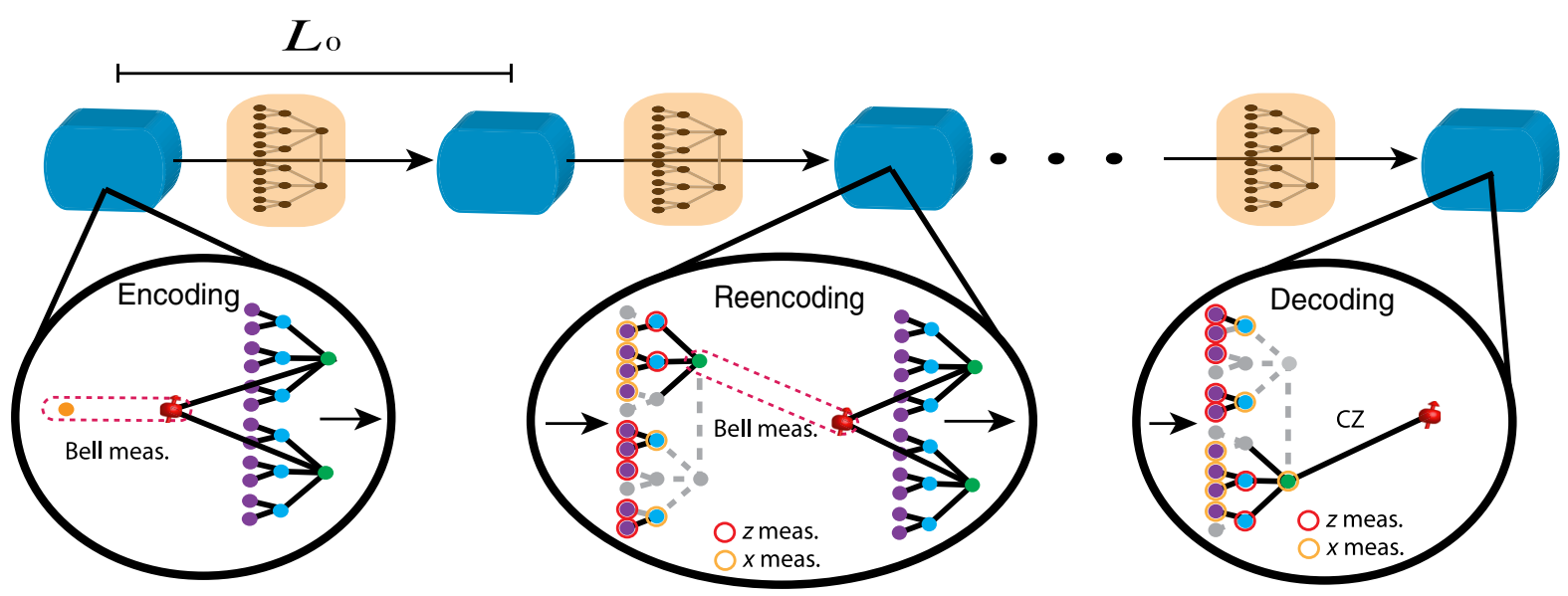

FIG. 1. Sketch of a one-way quantum repeater with photonic tree clusters. A message qubit (orange dot) is encoded through a Bell measurement (dashed box) with the root spin qubit of a photonic tree cluster. An example with a [2,3,2] tree is shown where dots correspond to photonic qubits and solid lines indicate correlations. The encoded qubit (depicted in the orange boxes) is sent a distance $L_{0}$ to the next repeater station. At the next repeater station, the qubit information is reencoded through a Bell measurement between one of the first-level photonic qubits and the root spin qubit of a new tree. The remaining qubits of the incoming tree are measured with single qubit $z$ (red circle) or $x$ (orange circle) measurements. The reencoding can succeed despite multiple photons being lost in transmission (gray dots and dashed lines). At the end station, the message qubit can be transferred to, e.g., a receiving spin qubit by means of a controlled-phase (Cz) gate.

qubit to be transmitted to the next repeater station (see Fig. 1). Photonic tree clusters have previously been considered as photonic memories to ensure efficient entanglement swapping in all-optical quantum repeaters [14,20]. Such all-optical approaches generate multiple photonic tree clusters at each repeater station potentially requiring kilometer-long delay lines and millions of single-photon sources per station [20]. Our approach circumvents this significant overhead by using strongly coupled quantum emitters with built-in nonlinearity. Specifically, in our approach, the photonic tree clusters required for the repeater can be generated with two memory qubits and one single-photon emitter per repeater station using repeated photon emissions [21]. In addition, correction of losses requires only a single Bell measurement independent of the size of the tree encoding. This constitutes a significant reduction in overhead as compared to, e.g., one-way quantum repeaters based on the quantum parity encoding $[11,12,15]$, which requires a number of two-qubit operations that scale linearly with the size of the encoding corresponding to hundreds of memory qubits per repeater station [7]. In comparison, the current approach can be implemented with only two spin systems per repeater station, as we outline below. We also discuss possible experimental implementations of our protocol based on state-of-the-art solid-state quantum emitters in nanophotonic structures in order to lay out a realistic path toward high bit rate, long-range quantum communication. Importantly, many of the required parameters for our protocol are not far from current state-of-the art performances, which together with the significant resource reduction compared to previous one-way protocols cements the experimental feasibility of our approach.

\section{QUANTUM REPEATER PROTOCOL}

The basic operation of the repeater is shown in Fig. 1. At each node, a multiphoton entangled state is generated and used to encode and transmit a message qubit to the next repeater station. Crucially, even if some of the photons are lost, the repeater can decode the logical qubit and reencode it, thereby correcting for photon loss before transmitting the message to the next station.

\section{A. Tree-cluster states}

In this work, we consider one-way quantum repeaters based on using tree-cluster states as error-correcting codes. Such tree-cluster states are illustrated in Fig. 2. We characterize the tree by a branching vector $\bar{t}=\left[b_{0}, b_{1}, \ldots, b_{d}\right]$, which specifies the connectivity of the tree as one moves from the root vertex [top node in Fig. 2(a)] through the $d$ levels of the tree. Tree-cluster states are obtained by associating a qubit with each vertex (see Fig. 1). Moreover, one further associates a stabilizer operator, $K_{i}=\sigma_{i}^{x} \otimes_{j \in \mathcal{N}(i)} \sigma_{j}^{z}$, that acts nontrivially on the vertex $i$ and its neighbors $\mathcal{N}(i)$. The treecluster state is the unique eigenstate with eigenvalue +1 of all stabilizer operators $K_{i}$.

As a specific, illustrative example, let us consider a [2,2] tree-cluster state [see Fig. 2(a)]. One can easily check that this 7-qubit state is given by

$$
\begin{aligned}
|\psi\rangle= & \frac{1}{2^{3 / 2}}\left(|0\rangle_{s}(|0\rangle|+,+\rangle+|1\rangle|-,-\rangle)^{\otimes 2}\right. \\
& \left.+|1\rangle_{s}(|0\rangle|+,+\rangle-|1\rangle|-,-\rangle)^{\otimes 2}\right) .
\end{aligned}
$$




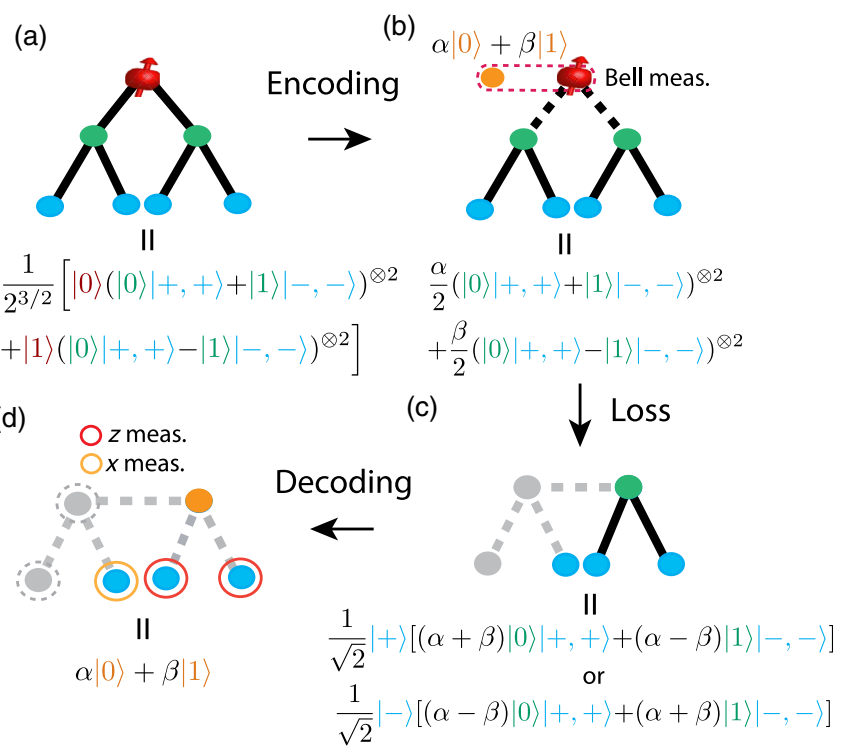

FIG. 2. Sketch of the principle behind counterfactual error correction in the tree-cluster encoding. A $[2,2]$ tree is considered for simplicity (a). A qubit $\alpha|0\rangle+\beta|1\rangle$ is encoded through a Bell measurement between this qubit and the root spin qubit (b). Loss of qubits (gray vertices) from one branch (c) still allows retrieving the encoded information by measuring at least one remaining second-level qubit in the $x$ basis and the second-level qubits of the intact branch in the $z$ basis (d) despite the fact that we do not get any information from (attempted) measurements on the lost photons (dashed circles).

Here, we have defined the states $| \pm\rangle=(|0\rangle \pm|1\rangle) / \sqrt{2}$, with $|0\rangle$ and $|1\rangle$ being the basis states of the qubits. Below we consider an implementation where the root qubit is represented by a stationary two-level spin system (spin qubits are denoted with subscript $s$ ) while the rest of the tree cluster state is represented by photons.

\section{B. Encoding the logical qubit}

Consider the situation where the message qubit, $\alpha|0\rangle_{s}+\beta|1\rangle_{s}$, is initially prepared in a second stationary qubit. To send this message qubit from the first station to the next repeater, one has to encode it into the state of the photons. This can be achieved by a simple teleportation process, which can be realized by a 2-qubit Bell measurement of the stationary qubits, i.e., the spin that stored the message qubit and the stationary root qubit of the treecluster state [see Fig. 2(b)].

For the above example of a $[2,2]$ tree, this prepares the state of the photons in

$$
\begin{aligned}
|\Psi\rangle= & \frac{\left(1+x_{1}\right) \alpha+\left(1-x_{1}\right) \beta}{4}(|0,+,+\rangle+|1,-,-\rangle)^{\otimes 2} \\
& +x_{2} \frac{\left(1-x_{1}\right) \alpha+\left(1+x_{1}\right) \beta}{4}(|0,+,+\rangle-|1,-,-\rangle)^{\otimes 2},
\end{aligned}
$$

where $x_{1}= \pm 1$ and $x_{2}= \pm 1$, depending on the four possible outcomes of the Bell measurement. The values of $x_{1}$ and $x_{2}$ are not important since they can eventually be corrected in the decoding step. In Fig. 2, we assume for concreteness that we have obtained the values $x_{1}=x_{2}=1$. Note that the quantum information of the message qubit is stored in a nonlocal form in the photonic degrees of freedom, and cannot be retrieved by observing, e.g., only a single photon.

\section{Photon loss and recovery of the message qubit}

After this encoding step, the photons are transmitted to the next repeater station. The specific encoding protects against transmission loss such that the effective transmission probability of the message qubit is significantly increased compared to the bare transmission of a single photon.

To illustrate the basic mechanism, we again consider the example of the $[2,2]$ encoding in Eq. (2). Already in this simple encoding, one can tolerate the loss of up to two photons in one of the two branches [see Fig. 2(c)]. To see this, it is instructive to consider how the state can be recovered and the quantum information retrieved. As a first step in the recovering process, one measures all the qubits in one of the two branches (in Fig. 2, we assume that the left branch is to be measured). Specifically, the first-level qubit is measured in the $z$ basis and the second-level qubits are measured in the $x$ basis. Note that the corresponding measurement outcomes are perfectly correlated, such that only two sets of outcomes for the three measurements are possible. This is crucial, as it allows us to infer the outcome from each of the three measurements, even if two of those qubits are lost. This measurements projects the state of the qubits in the remaining branch [right branch in Fig 2(c)] into the state

$$
\begin{aligned}
& \frac{\left(1+x_{1}\right) \alpha+\left(1-x_{1}\right) \beta}{2 \sqrt{2}}(|0,+,+\rangle+|1,-,-\rangle) \\
& +x_{2} x_{3} \frac{\left(1-x_{1}\right) \alpha+\left(1+x_{1}\right) \beta}{2 \sqrt{2}}(|0,+,+\rangle-|1,-,-\rangle),
\end{aligned}
$$

where $x_{3}= \pm 1$, depending on the measurement outcome. This branch now contains the entire encoded quantum information. It can be retrieved by measuring the two second-level qubits in the $z$ basis [see Fig. 2(d)]. Simple algebra shows that this prepares the remaining first-level qubit in the state $\alpha|0\rangle+\beta|1\rangle$ (up to known Pauli corrections that only depend on the obtained measurement outcomes $x_{i}$ ). This simple analysis shows that the retrieval of the message qubit from a $[2,2]$ tree is possible as long as one branch is not corrupted and not more than two qubits of the other branch are lost, illustrating the basic principle allowing for correction of photon loss. Increasing the tree 
depth (length of $\bar{t}$ ) and the number of branches increases the robustness of the encoding by the same principle [19].

\section{Reencoding and repetition}

The goal of the repeater station is to reencode the retrieved qubit in a new tree. This can be achieved in complete analogy to the encoding of the message qubit at the sending station: first a new tree cluster of photons is generated, with a stationary spin serving as the root qubit (see Sec. III A), followed by a Bell measurement between the message qubit and the root qubit.

Above we described how to recover the qubit into a photonic qubit in the highest level of the tree. In the reencoding procedure the goal is instead to perform a Bell measurement between the encoded qubit and the root qubit of a new tree. In analogy with the procedure above, this reencoding simply requires a Bell measurement between one of the first-level photons of the encoded tree cluster and the root qubit along with measurement of all other qubits in the same bases as above. Note that the order of the measurements is not important in the above recovery scheme. In practice, this allows us to reencode the quantum information at each repeater station without prior knowledge about which qubit was lost. Specifically, one can first attempt a Bell measurement between one of the first-level qubits and the root qubit of the new tree. If this measurement is successful, one can teleport the encoded quantum information into the new tree by measuring all connected qubits in the $z$ basis. Some of these measurements may turn out to be unsuccessful because the qubits were lost in transmission. In these cases, the corresponding measurement outcome is inferred through measurements on qubits in the next level of the corresponding branch, in complete analogy to the example above. If the first Bell measurement is unsuccessful itself (because the corresponding photon was lost in transmission), then the value of a $z$ measurement can be inferred instead (via measurement of the next-level qubits), and a Bell measurement can be attempted with another first-level qubit. In order not to perturb the root qubit of the new tree in a failed attempt of a Bell measurement, special care must be given to the implementation of the measurement, as described below. Specifically, the message qubit should first be transferred to an auxiliary spin qubit by means of a spin-photon controlled-phase gate (CZ gate) and then encoded into the new tree cluster with a deterministic Bell measurement between the auxiliary spin qubit and the root spin qubit (see Fig. 4).

The reencoding and transmission continues down the repeater chain until the encoded message qubit arrives at the end node. There the message qubit can be either transferred to a stationary spin in a similar fashion as in the repetition step (see Fig. 1) or directly measured (without first transferring the information to a receiving spin qubit) by appropriate measurements of the photons of the encoded tree.

\section{EXPERIMENTAL IMPLEMENTATION}

The key requirements for an implementation of the above protocol are the ability to generate tree-cluster states of photons, realize Bell measurements between stationary spins and photonic qubits, and perform measurements of the photons in the $x$ and $z$ basis.

\section{A. Photonic tree generation}

We propose to generate the photonic tree cluster states using a light-matter interface illustrated in Fig. 4. It consists of stationary memory spins and one spin which is coupled to the light field. The latter is used to generate photons by selectively coupling a ground state $|1\rangle_{s}$ to an excited $|e\rangle_{s}$ via the optical field in a one-sided cavity. We are considering a time-bin representation of the photonic qubits. In this representation, the presence of a single photon in one of two nonoverlapping spatiotemporal modes represents the two qubit states $|0\rangle$ and $|1\rangle$. The main reason for using this time-bin representation is that it allows us to detect errors stemming from photon loss. This could also be obtained with a polarization representation, but the time-bin representation is better suited for transmittance through optical fibers, which typically disturb the polarization state.

Recent work [21] showed that sequential excitation of the quantum emitter, together with controlled-phase gates between the emitter and the memory spins, allows us to deterministically generate an arbitrary photonic tree-cluster state. In particular, a tree-cluster state of depth $d+1$ requires only $d$ memory spin systems while the number of necessary spin-spin CZ gates scales polynomially with the number of branches at each level [21]. In what follows, we show that tree-cluster states of depth 3 are sufficient for transmission distances up to $1000 \mathrm{~km}$ (assuming telecom frequencies) and consequently only two memory spin systems and a single quantum emitter are necessary for the generation of such states.

The generation of a tree-cluster state with depth 3 is sketched in Fig. 3. In the first step [Fig. 3(a)], CZ gates are applied between the two memory spins and between the emitter and one memory spin. The spin of the quantum emitter acts as a second-level qubit in the tree, and all thirdlevel photonic qubits of the subbranch are emitted through repeated excitation followed by spontaneous emission, as shown in Fig. 3(b). The second-level qubit is then mapped to a photonic qubit using the auxiliary level of the emitter, which also detaches the emitter from the preliminary treecluster state. This step is repeated until all second- and third-level photons of the branch have been emitted. The spin of the second memory qubit is then first swapped to the spin of the emitter by means of two $\mathrm{CZ}$ gates and subsequently swapped to a photonic qubit [Fig. 3(c)]. 
(a)

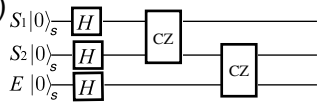

(b)$$
\text { (b) }
$$

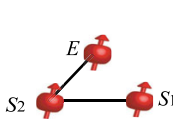

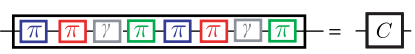

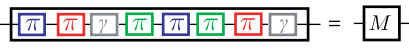
$E-C, C, C M$
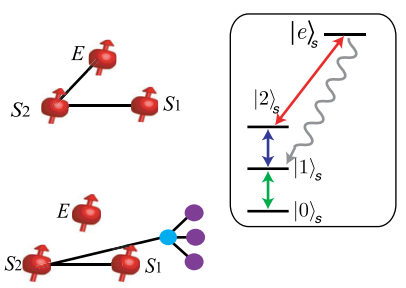

(c)
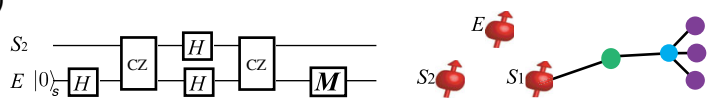

FIG. 3. Generation of photonic tree-cluster states. Two memory spin systems $\left(S_{1}, S_{2}\right)$ and a quantum emitter $(E)$ are all prepared in state $|+\rangle_{s}$, and CZ gates entangle the spins as shown in (a). In the second step (b), all third-level photons (purple dots) of one sub-branch of the tree cluster are emitted (operation $C$ ) followed by the emission of the corresponding second-level photon (blue dot), which detaches the emitter from the preliminary tree cluster (operation $M$ ). Operations $M$ and $C$ can be implemented using a sequence of $\pi$ pulses on various transitions in the emitter, as indicated by the color code in (b) referring to the color of the transitions in the emitter level diagram shown in the inset. Here driven transitions are indicated by solid lines, whereas decay $(\gamma)$ is represented by the wiggle line. To emit more second-level qubits, $E$ is again prepared in $|+\rangle_{s}$ followed by a CZ gate with $S_{2}$ and step (b) is repeated. In step (c), the state of the memory spin is first swapped to the emitter and then emitted as the first-level photon of the branch. Here the emission of the first-level photon is achieved by an operation $\boldsymbol{M}$, which is similar to $M$ except that the red $\pi$ pulse and subsequent decay is replaced by a weak driving of the $|2\rangle_{s}-|e\rangle_{s}$ transition to ensure that the photons have a narrow bandwidth. The steps are then repeated until the entire tree has been emitted.

This completes the emission of one branch of the photonic tree-cluster state, and the procedure can then be repeated to output the entire state. We note that this generation procedure, in principle, requires additional Hadamard gates on the third-level photons to fit the stabilizer description introduced earlier. This is, however, not necessary since it is sufficient to simply rotate the measurement basis of these photons in the reencoding and decoding steps.

A drawback of this generation scheme is that the photons will be emitted such that the first-level qubit of a branch is emitted last. As previously described, the presence or absence of a first-level photon determines the measurement basis of the corresponding branch at a repeater station. It is therefore necessary to delay the photons of each branch to enable measuring the first-level qubit first. The length of this delay will depend on the emission rate of the emitters and the number of photons per branch, but is generally modest and implementable in optical fiber delays. We discuss this issue in more detail below.

\section{B. Bell measurement}

As described above, the reencoding at the repeater stations requires a successful Bell measurement between
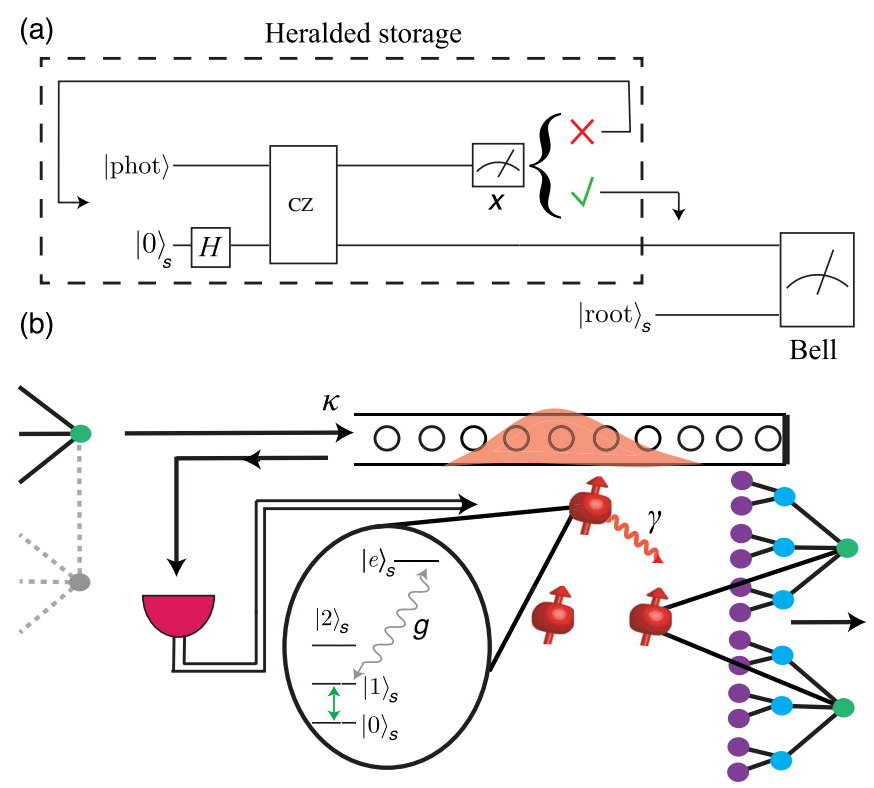

FIG. 4. The basic elements of the reencoding operation at the repeater stations. (a) The reencoding is performed in a loss tolerant manner by first performing a heralded storage of the message qubit in an auxiliary memory spin (dashed box). This is obtained through a spin-photon controlled-phase (CZ) gate with a first-level photon of the encoded tree. The transfer is heralded by the detection of the photon in the $x$ basis. If unsuccessful (photon loss), the auxiliary spin is initialized and the operation is tried again with another first-level photon. Once the storage is successful, a deterministic Bell measurement is performed between the auxiliary spin and the root spin qubit of the new tree cluster, thereby completing the reencoding operation. (b) The experimental implementation of the reencoding operation. The spin-photon CZ gate is performed by reflecting the photonic qubit off a one-sided cavity (shown as a photonic crystal cavity) strongly coupled to a quantum emitter, as described in the text. The parameters $g, \gamma$, and $\kappa$ are the single-photon Rabi frequency of the optical transition, the spontaneous emission rate of the emitter, and the decay rate of the cavity field, respectively. The same physical setup is used to generate the tree-cluster state requiring an extra spin qubit and one auxiliary level $\left(|2\rangle_{s}\right)$ in the quantum emitter (see Fig. 3).

one of the first-level qubits in the encoded tree cluster and the root qubit of the new tree cluster. It is crucial that this Bell measurement is designed with a built-in error detection: if a Bell measurement is attempted with a lost first-level qubit, the measurement should abort without perturbing the root qubit. Otherwise, a new tree cluster has to be generated after each failed attempt.

The setup required to generate the tree-cluster states [Fig. 3(a)] conveniently also allows for such an operation. While one of the stationary memory qubits represents the root qubit, the spin coupled to a one-sided cavity is used for heralded storage of the message qubit through a spinphoton CZ gate (see below) [22-25]. Importantly, the success of the storage is conditioned on subsequently 
detecting the photon in the $x$ basis [see Fig. 4(a)]. When a storage attempt is unsuccessful due to the loss of the photonic qubit, the auxiliary spin qubit is simply reinitialized and a new attempt is made with another first-level qubit. The root spin qubit of the new tree cluster is completely unaffected by this. Once the storage is successful, a Bell measurement between the auxiliary spin system and the spin system containing the root qubit of the new tree is performed using deterministic entangling gates between the two spin systems, concluding the reencoding step.

To perform the cavity-mediated spin-photon CZ gate, we assume the auxiliary spin to initially be prepared in ground state $|0\rangle_{s}$. For a time-bin encoded photonic qubit, the early half of the wave packet corresponding to qubit state $|0\rangle$ is first reflected off the cavity. In the ideal limit, the photon will be reflected with a $\pi$-phase shift from the cavity. Now the transformation $|0\rangle_{s} \rightarrow\left(|0\rangle_{s}+|1\rangle_{s}\right) / \sqrt{2}$ is performed on the emitter before the late half of the photon wave packet, corresponding to qubit state $|1\rangle$, is reflected off the cavity. If the auxiliary spin system is in state $|1\rangle_{s}\left(|0\rangle_{s}\right)$, the photon gets reflected without (with) a $\pi$-phase shift in the limit of strong light-matter interaction $C \gg 1$, characterized by the cooperativity $C=g^{2} /(\kappa \gamma)$. Here $g$ is the single-photon Rabi frequency of the cavity-mediated $|1\rangle \leftrightarrow|e\rangle$ transition, $\kappa$ is the decay rate of the cavity, and $\gamma$ is the free-space spontaneous emission rate of the excited level. Up to a global phase, this amounts to a CZ gate between the photonic time-bin qubit and a qubit in the ground states of the auxiliary spin system initially prepared in $\left(|0\rangle_{s}+|1\rangle_{s}\right) / \sqrt{2}$. The details of the gate interaction and main errors are described in the Supplemental Material [26]. The success of the gate is conditioned on subsequently detecting the photon in the $x$ basis, which boosts the fidelity. We find that if the intracavity losses are tuned to be on the order of $1 / C$, then a spin-photon cooperativity of $C=100$ is sufficient to ensure an error $\lesssim 10^{-4}$ and a success probability $\sim 0.99 \eta_{d}$, where $\eta_{d}$ is the efficiency of the photon detection.

In the above estimate, we have assumed that reflection of photons into the detector from, e.g., imperfect mode matching is negligible. Such events directly translate into an error since they correspond to operation without any spin-photon interaction. Careful engineering of the mode profile and additional filtering of, e.g., uncoupled polarization modes must therefore be employed to suppress such reflections to the desired error level. We note, however, that a mode-matching efficiency of $99 \%$ is sufficient to ensure an error of $10^{-4}$ [26]. Furthermore, we have assumed that the frequency width of the first-level photons is narrow enough to neglect errors from the finite bandwidth of the Purcell-enhanced emitter. For $C=100$, this would require the first-level photons to have a frequency width $\sim \gamma$ to have errors $\lesssim 10^{-4}$ [26]. Weak driving from an auxiliary level $|2\rangle_{s}$ to $|e\rangle_{s}$ [see inset in Fig. 3(a)] allows us to tune the emission time of the first-level photons to achieve this [27] (operation $\boldsymbol{M}$ in Fig. 4).

We note that depending on the success of the spinphoton Bell measurement, the measurement basis of the qubits in the corresponding branch of the encoded treecluster states must be adjusted. If a first-level qubit is lost (detected), the qubits in the corresponding branch should be measured in the $x(z)$ basis for the tree-cluster states generated as described above.

\section{Photon measurement}

With the considered time-bin encoding, measurements of photons in the $z$ basis require only time-resolved detection. Measurements in the $x$ basis, on the other hand, are more demanding. In particular, a deterministic $x$-basis measurement requires fast optical switching and delay lines. Our analysis shows that gigahertz optical switching rates will be required to ensure tree-generation rates in the megahertz regime (see below). Such switching rates can be exceeded with schemes based on subnanosecond phase control in Mach-Zehnder interferometers via electro-optic modulation. Such integrated devices have been demonstrated for a variety of material platforms [28-33]. Toward a scalable, small footprint implementation, we therefore propose an on-chip photonic circuit based on switching via electrooptic modulation in cascaded Mach-Zehnder interferometers as shown in Fig. 5 and discussed in the Supplemental Material, where we also outline an integrated on-chip setup for the repeater stations [26].

\section{Other experimental requirements}

So far we have discussed the optical interface required to achieve the successful operation of the repeater. In order to be able to make a realistic estimate of the achievable communication rate, we now discuss the concrete requirement for

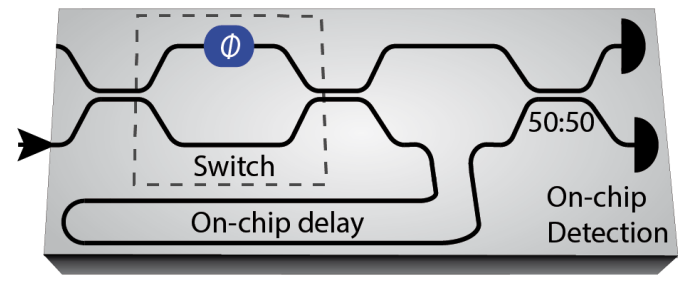

FIG. 5. Illustration of an on-chip photonic circuit for performing $z$ - and $x$-basis measurements with integrated single-photon detectors. Mach-Zehnder interferometers with fast switching rate, for example, based on electro-optic switching [28-33], are assumed to switch fully between the top and bottom path (labeled switch) by controlling the relative phase of the two interferometer $\operatorname{arms}(\phi)$. For a $z$-basis measurement, the photons are guided directly to the detectors for time-resolved recording. For an $x$-basis measurement, the switch guides the first wave package of the time-bin encoding to the delay line and the second to the short arm. For first-level photons the delay needs to be longer and is replaced with a fiber delay line [26]. 

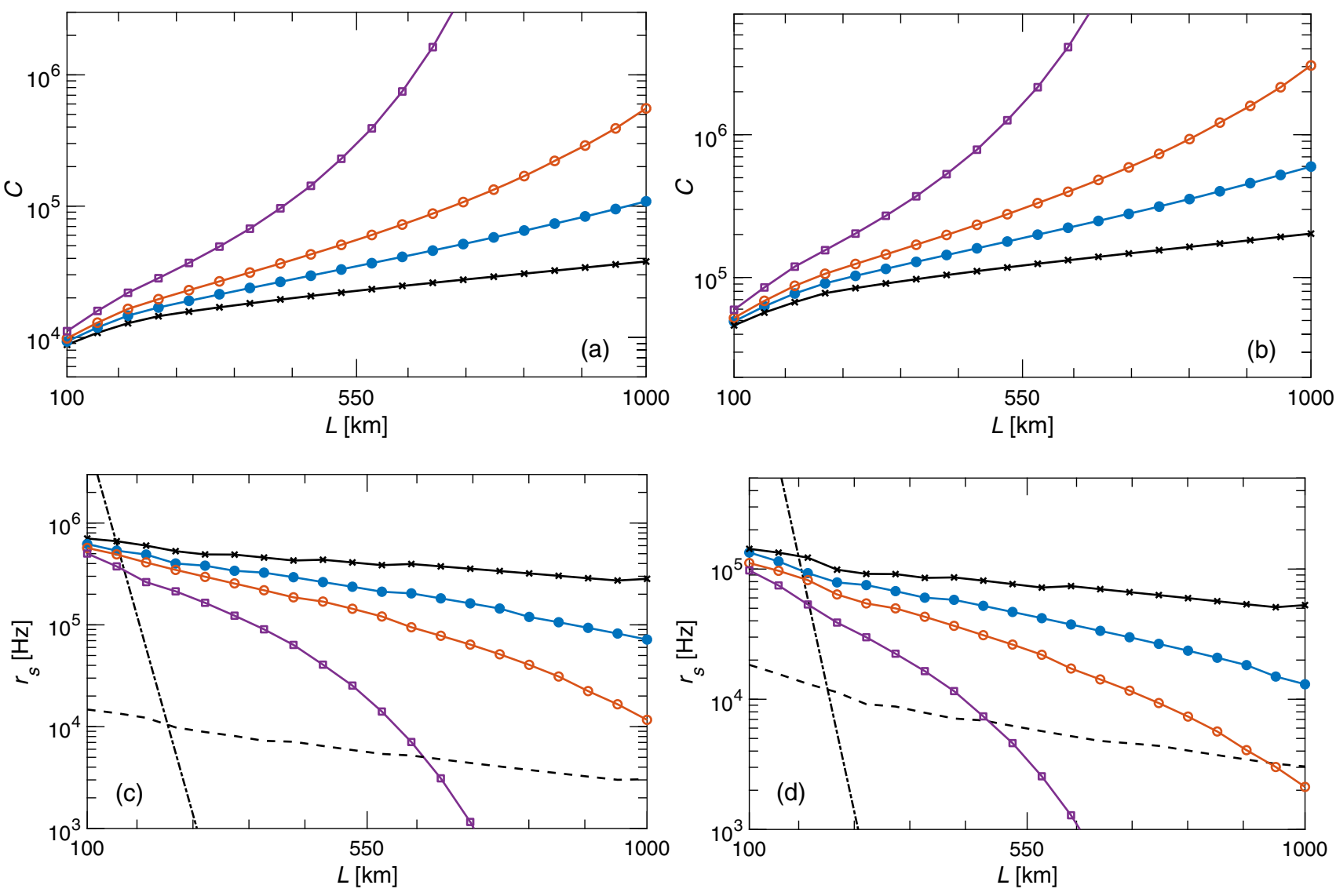

FIG. 6. (a),(b) Minimized cost parameter $C$ as a function of distance $L$ assuming spin entangling gate times of $\tau_{\mathrm{CZ}}=10 \tau_{\mathrm{ph}}$ (a) and $\tau_{\mathrm{CZ}}=100 \tau_{\mathrm{ph}}$ (b) for tree-cluster state generation. The markers correspond to times symbol, $\epsilon_{r}=0.1 \%$; filled circle, $\epsilon_{r}=0.3 \%$; circle, $\epsilon_{r}=0.5 \%$; square, $\epsilon_{r}=0.1 \%$. Here, $\epsilon_{r}$ is the error probability of the reencoding operation at the repeater stations. (c),(d) Corresponding secret bit rate assuming a photon emission time of $\tau_{\mathrm{ph}}=1 \mathrm{~ns}$ and gate time of $\tau_{\mathrm{CZ}}=10 \mathrm{~ns}(\mathrm{c})$ and $\tau_{\mathrm{CZ}}=100 \mathrm{~ns}$ (d). We have restricted the minimization to trees with $n \leq 300$ photons and repeater station spacings $\geq 1 \mathrm{~km}$. We have assumed a detection efficiency of $\eta_{d}=95 \%$. For comparison, the secret bit rate of a two-way repeater (dashed line) with similar resources (see main text) is also plotted together with the rate of direct transmission (dot-dashed line) assuming a 1 GHz single-photon source.

two specific physical systems, quantum dots and color centers in diamond. One of the practical requirements in reducing the photon loss is a highly efficient coupling for the cavity to an optical fiber, e.g., using tapered optical fibers [34-36]. The collection efficiency ( $\beta$ factor) of the emitted photons to a cavity or alternatively a waveguide needs to be high. Quantum dots in waveguides have already demonstrated collection efficiencies of $\beta>98 \%$ [37], which is compatible with the efficiency $\eta_{d}=0.95$ assumed in our resource analysis below (see Fig. 6). In addition, coupling to photonic nanostructures may also decrease the photon emission time through the Purcell enhancement, and photon emission times of $\sim 100 \mathrm{ps}$ are feasible with solid-state emitters such as quantum dots [38] and diamond color centers [39].

Finally, spin-spin CZ gates are required for both the treegeneration and the reencoding operation. Fast spin-spin gates ( $\sim 10 \mathrm{~ns})$ could be performed in stacked quantum dots $[40,41]$ while somewhat slower gates can be performed between electron and nuclear spins for nitrogen-vacancy
(NV) or silicon-vacancy (SiV) centers in diamond through magnetic dipolar interaction [42]. For the latter, gate times on the order of $100 \mathrm{~ns}$ are feasible with $\mathrm{SiV}$ systems using nearby nuclei with strong $(>1 \mathrm{MHz})$ hyperfine interactions. Alternatively, fast gates could also be implemented using photon-mediated gates between different emitters [43]. This involves two auxiliary spin qubits for parity measurements and can be made error proof against photon loss errors at the expense of a slight decrease in success probability. For the gate in question [43], a $\beta$ factor of $\sim 0.99$ would give a success probability of $\sim 99 \%$ and a heralded error of $\sim 0.1 \%$ o. Such probabilisitic spin-spin gates will, however, decrease the rate of the repeater when used in the reencoding step at the repeater stations. The reason being that the reencoding involves the (unprotected) root qubit of the new tree. Considering a distance of $1000 \mathrm{~km}$ where $\sim 400$ repeater stations are needed (see optimization below), a success probability of $99 \%$ would result in a rate that is $\sim 2 \%$ of the rate for a deterministic gate. Using probabilistic gates in the tree generation steps is 
of less concern since heralding techniques can be employed and the majority of the necessary gates will involve the redundant qubits of the tree encoding, which are somewhat loss tolerant.

The details of the time budget for the generation of the tree-cluster states and length of the necessary delay line is detailed in the Supplemental Material [26]. We find that the photonic tree-cluster states can be emitted within $\sim 1 \mu \mathrm{s}$ $(\sim 7 \mu \mathrm{s})$ assuming Purcell-enhanced photon emission lifetime of about $100 \mathrm{ps}[38,39]$ and spin-spin CZ gate times of $10 \mathrm{~ns}$ (100 ns). The spin qubits need to stay highly coherent for these timescales, which for $\mathrm{SiV}$ and NV systems can be achieved using nearby nuclear spins [44] or operating at low temperatures [45]. For quantum dots, dynamical decoupling [46] or coupling to a nuclear spin memory [47] may be employed to increase coherence times motivating further development of such techniques. For the above generation times, delay lines of maximum length $\sim 68 \mathrm{~m}(\sim 374 \mathrm{~m})$ at the repeater stations are required to ensure the right detection order of the photons (see Sec. III A). At telecom frequencies such a delay line would have a transmission above 99\% ( 98\%), which can be integrated into the overall detection efficiency $\eta_{d}$.

\section{REPEATER PERFORMANCE}

The above analysis outlined all necessary operations and general hardware considerations of the repeater. Importantly, we have shown that only a single successful Bell measurement is needed at the reencoding step and that this can be implemented in a loss-tolerant manner using two spin systems. Furthermore, we have outlined how the photonic tree clusters may be generated requiring in total only two qubit spin systems per repeater station in addition to the quantum emitter. We now proceed by estimating the performance of the repeater in terms of the maximum quantum bit rate for given distances.

The transmission probability of a message qubit through the entire repeater chain will be

$$
p_{\text {trans }}=\eta_{e}^{m+1},
$$

where $m$ is the number of equally spaced repeater stations between the start and end stations, and $\eta_{e}$ is the transmission probability of the encoded quantum information between repeater stations. The encoded transmission probability depends on the specific tree encoding, the bare transmission probability $\eta$ of a single photon between repeater stations, and the detection efficiency of the photon detectors $\eta_{d}$. Note that in-out coupling efficiency and any frequency conversion efficiency that may be required to transduce to the telecom band can be directly included in $\eta_{d}$.

For a tree-cluster encoding with branching vector $\vec{t}=\left[b_{0}, b_{1}, \ldots, b_{d}\right], \eta_{e}$ is given by the recursive formula [19]

$$
\eta_{e}=\left[\left(1-\mu+\mu R_{1}\right)^{b_{0}}-\left(\mu R_{1}\right)^{b_{0}}\right]\left(1-\mu+\mu R_{2}\right)^{b_{1}},
$$

where

$$
R_{k}=1-\left[1-(1-\mu)\left(1-\mu+\mu R_{k+2}\right)^{b_{k+1}}\right]^{b_{k}},
$$

with $R_{d+1}=0, b_{d+1}=0$, and $\mu=1-\eta \eta_{d}$. Here, $R_{k}$ is the probability of having a successful indirect $z$ measurement of any given qubit in the $k$ th level of the tree. Consequently, the total probability of a successful $z$ measurement of a $k$ th level qubit (direct or indirect) is $1-\mu+\mu R_{k}$. For a fiber-based implementation, the bare transmission will be $\eta=\exp \left(-L_{0} / L_{\text {att }}\right)$, where $L_{0}$ is the distance between the repeater stations and $L_{\text {att }}=20 \mathrm{~km}$ is the attenuation length of the optical fiber assuming that efficient frequency conversion to the telecom band is implemented.

The relative simplicity of the encoding in tree-cluster states comes with the penalty that it is not able to correct arbitrary errors as opposed to other codes considered for one-way repeaters. It is clear that an error on the qubits participating in the reencoding Bell measurement will map into an error on the encoded message qubit. However, there is some robustness against errors due to the large redundancy of information encoded in the tree [14]. As discussed below, this leads to an error rate of the encoded qubits, which is only a few times the error rate of the individual qubits.

To quantify the performance of the tree repeater in the presence of operational errors, we consider the secret bit fraction $f$ of the transmitted qubits, which can be estimated in the asymptotic limit of infinitely long keys assuming perfect classical error correction. Assuming quantum key distribution is performed using a six-state variant of the BB84 protocol we have that [17]

$$
f=1-h(Q)-Q-(1-Q) h\left(\frac{1-3 Q / 2}{1-Q}\right),
$$

where $Q=2 \epsilon_{\text {trans }} / 3$ is the qubit error rate of the transmitted bits and $h(x)=-x \log _{2}(x)-(1-x) \log _{2}(1-x)$ is the binary entropy. We have assumed a (worst-case) scenario where the noise on the transmitted bits are described by a single qubit depolarizing channel of the form

$\Lambda(\hat{\rho})=\left(1-\epsilon_{\text {trans }}\right) \hat{\rho}+\frac{\epsilon_{\text {trans }}}{3}\left(\sigma^{x} \hat{\rho} \sigma^{x}+\sigma^{y} \hat{\rho} \sigma^{y}+\sigma^{z} \hat{\rho} \sigma^{z}\right)$,

where $\hat{\rho}$ is the density matrix of the (pure) message qubit and $\sigma_{i}^{x, y, z}$ are the Pauli matrices. The final error probability of the transmitted message qubit is $\epsilon_{\text {trans }}$. For a repeater with $m$ repeater stations, it is estimated as $\epsilon_{\text {trans }}=1-(1-$ $\left.\epsilon_{r}\right)^{m+1} \approx(m+1) \epsilon_{r}$ for $m \epsilon_{r} \ll 1$, where $\epsilon_{r}$ is the error probability of the reencoding step at the repeater stations. Note that $f$ is negative for $Q \gtrsim 12.61 \%$ reflecting that it is no longer possible to extract any secret bits from the 
transmitted qubits since they are too noisy for privacy amplification. Since the tree encoding is not able to correct the errors, this will eventually limit the distance to $L \sim 0.13 L_{0} / \epsilon_{r}$.

\section{A. Optimization of repeater performance}

In order to asses the performance of the repeater, we perform a numerical optimization of the number of repeater stations $(m)$ and the encoding tree $(\vec{t})$ for a given distance and error $\left(\epsilon_{r}\right)$ to find the highest possible secret bit rate. We assume that the local repetition rate $r_{0}$ is set by the emission time of the photonic tree-cluster states. For realistic parameters (see below), this will be determined by the emission time of a photonic qubit $\left(\tau_{\mathrm{ph}}\right)$ and the gate time $\left(\tau_{\mathrm{CZ}}\right)$ of spin-spin gates. For a specific tree encoding $\left(\vec{t}=\left[b_{0}, b_{1}, \ldots, b_{d}\right]\right)$ we estimate the generation time as

$$
\begin{aligned}
\frac{1}{r_{0}} & \sim b_{0}\left[100+b_{1}\left(1+b_{2}\left(1+\cdots b_{d-1}\left(1+b_{d}\right) \cdots\right)\right)\right] \tau_{\mathrm{ph}} \\
& +b_{0}\left[3+b_{1}\left(1+b_{2}\left(1+\cdots b_{d-2}\left(1+b_{d-1}\right) \cdots\right)\right)\right] \tau_{\mathrm{CZ}} .
\end{aligned}
$$

Note that we assume the emission time of the first-level photons to be $100 \tau_{\mathrm{ph}}$ to have errors $\sim 10^{-4}$ in the scattering gate of the reencoding step (see above). In addition, three spin-spin entangling gates are needed for the creation of the first-level qubits. We then seek to minimize the (dimensionless) cost parameter,

$$
C=\frac{1}{r_{0} f p_{\text {trans }}} \frac{m L_{\text {att }}}{\tau_{\mathrm{ph}} L}
$$

The inverse cost parameter can be viewed as the secret key rate in units of the photonic qubit emission time per repeater station per attenuation length for a given total distance, $L$. In the optimization, we enforce a maximum of the number of photons in the encoding of $n=300$ and require that the repeater stations are never placed closer than $1 \mathrm{~km}$ apart. The results of the optimizations are shown in Fig. 6.

It is clear that as the operational errors increase, the repeater performs worse since the tree encoding is not fault tolerant with respect to depolarizing errors. Nonetheless, for $\epsilon_{r} \lesssim 0.1 \%$, it is still possible to reach high secret bit rates since the repetition rate is determined by the local repetition rate, which can be in the megahertz regime. Specifically, with a photon emission time of $\tau_{\mathrm{ph}}=1 \mathrm{~ns}$ and gate time of $\tau_{\mathrm{CZ}}=10 \mathrm{~ns}$, a secret bit rate of $\sim 70 \mathrm{kHz}$ over $1000 \mathrm{~km}$ is possible with a repeater station spacing of $2.6 \mathrm{~km}$, a detection efficiency of $\eta_{d}=95 \%$, a reencoding error of $\epsilon_{r}=0.3 \%$, and using $[4,14,4]$ trees of 285 photons. For more modest gate times of $\tau_{\mathrm{CZ}}=100 \mathrm{~ns}$, a secret bit rate of $\sim 13 \mathrm{kHz}$ over $1000 \mathrm{~km}$ for the same parameters can be achieved (see above and Supplemental
Material [26] for a justification of these numbers for a concrete physical realization).

\section{B. Logical errors}

We have assumed a generic reencoding error $\epsilon_{r}$ in our optimization above. This encoding error will, in general, be determined by errors from both the generation of the photonic tree clusters and the reencoding step. In the optimization, we assumed a fixed $\epsilon_{r}$ and optimized the tree encoding for the given distances (see Supplemental Material [26]). One could imagine that $\epsilon_{r}$ will depend on the size of the encoding. To investigate this, we consider single qubit depolarizing channels of the form in Eq. (8) acting on all qubits in the encoding. We can then estimate the single qubit error probability $\epsilon$ that will result in a given reencoding error probability $\epsilon_{r}$ for a specific tree encoding as detailed in the Supplemental Material [26]. The tree encodings are remarkably robust to errors even in the presence of loss and we find that $\epsilon_{r} / \epsilon \approx 3$ for tree encodings and loss corresponding to the optimization in Fig. 6 except for the high error $\left(\epsilon_{r}=0.1 \%\right)$ optimization, where we find that $\epsilon_{r} / \epsilon \approx 4-5$. Notably, we do not find any significant dependence of $\epsilon_{r}$ on the different tree encodings. This is consistent with the errors of the two qubits (first-level qubit and root qubit) participating in the Bell measurement dominating the reencoding error.

\section{Comparison to other approaches}

The proposed repeater compares favorably to previously proposed one-way repeater protocols [12-14,16,18] (see Table 1 in the Supplemental Material [26]). It enables similar secret key rates for roughly the same error parameters and detection efficiencies. The key advantage of this repeater, however, is that it requires substantially less resources per repeater station than any of the previous protocols. In particular, the number of spin qubits per repeater station is 2 orders of magnitude lower than the matter based protocol in Ref. [12] and the large overhead of single-photon sources for linear optics protocols [14] is circumvented. The resources for the latter may be reduced by generating the photon cluster states as proposed in Ref. [21]. Nonetheless, the size of the encoding is still more than an order of magnitude larger than for our protocol. We obtain this reduction by directly encoding the message qubit in a tree-cluster state and using the spin-photon interface for near-deterministic reencoding operations as opposed to swapping entanglement with probabilistic linear optics Bell measurements. Compared to other one-way repeaters, the proposed repeater is, however, not fault tolerant and the tolerable error level therefore decreases with the distance. This is simply a consequence of the buildup of error with each reencoding operation. Thus, and order of magnitude decrease in error roughly corresponds to an order of magnitude increase in distance (e.g., distances in the range $10^{3}-10^{4} \mathrm{~km}$ would be achievable 
with an error rate of $10^{-4}-10^{-5}$ and tree-cluster states of depth 3). It might be possible to remedy this effect by incorporating error correcting for logical errors at the expense of a few additional spins at each station. A full investigation of this is, however, beyond the scope of this article.

We have also compared the repeater to a two-way quantum repeater allowing for parallel entanglement generation attempts using the same total number of spin qubits as our one-way repeater (see Supplemental Material [26]). We have assumed deterministic noise-free entanglement swapping and noise-free entanglement generation using a two-photon interference scheme [48]. This provides a crude comparison with standard two-way repeaters. As shown in Fig. 6(b), our one-way repeater reaches key rates orders of magnitude higher than the two-way repeater due to the higher local repetition rate. Furthermore, this advantage is achieved without the need for long coherence times. For the two-way repeater, orders of magnitude larger coherence times (miliseconds to seconds) are required. Note that Ref. [7] contains an extended comparison between one-way and two-way repeaters also showing the advantage of the former in the low noise limit. The comparison performed so far has been made in terms of communication rate per qubit by requiring the two repeater approaches to have the same total number of qubits. In practice, other factors may also be relevant for the comparison. In particular, the number of repeater stations is about an order of magnitude larger for the one-way repeater and the initial cost of establishing a quantum repeater chain is thus likely to be higher with the present approach. This is, however, compensated by a much higher communication rate resulting in a better rate per qubit.

\section{CONCLUSION AND DISCUSSION}

We have proposed a novel one-way quantum repeater based on photonic tree-cluster states. The repeater enables secret bit rates $\sim 70 \mathrm{kHz}(\sim 13 \mathrm{kHz})$ over a distance of $1000 \mathrm{~km}$ assuming gigahertz single-photon emission rates and spin-spin entangling gate times of $10 \mathrm{~ns}(100 \mathrm{~ns})$. We have demonstrated how both the error correction and the generation of the tree-cluster states at the repeater stations can be performed with a minimum number of spin systems. Specifically, we have outlined a repeater setup that requires only a single quantum emitter and two memory spin qubits per station. As compared to the daunting requirement for realizing previously proposed one-way quantum repeaters, this places our proposal within experimental reach of current technologies.

Solid-state systems such as quantum dots and diamond defects are promising hardware candidates. Single-photon emission rates exceeding gigahertz have already been achieved $[38,39]$ together with efficient coupling to nanophotonic waveguides and cavities [42,49-52]. The spinspin gates required for the repeater may be mediated through tunneling in quantum dots [40,53,54] or nuclear-electron spin coupling for diamond defects [42]. While many of the key elements necessary for this proposal have already been demonstrated, additional engineering of the platforms will be required in order to reach the required photon collection efficiencies and error level of the gates. Importantly, the proposed implementations based on stateof-the-art solid-state emitters appear capable of reaching those demanding metrics. Notably, both high cooperativity and combined detection and in-out coupling efficiencies above $90 \%$ have recently been reported with a SiV defect center coupled to a nanophotonic cavity [55]. For quantum dots, chip-to-fiber coupling efficiencies exceeding $80 \%$ have been reported [36], which could readily be improved further. High cooperativity has also recently been demonstrated by coupling a quantum dot to a microcavity [56]. The current state of the art is thus not far from the required performance of this protocol. In addition, our protocol outperforms direct transmission already at overall detection efficiencies of $85 \%$, and for slightly higher efficiencies, error levels $>0.1 \%$ can be tolerated, as shown in the Supplemental Material [26]. Both efficient frequency conversion to the telecom $\mathrm{C}$ band (where low-loss optical fibers exist) and fast optical switching are necessary to achieve long communication distances. While this remains a challenge, there has been impressive progress on highefficiency frequency conversion [57-59] and fast optical switching [60] with clear routes toward further improving the performance. An alternative strategy is to develop quantum dots emitting directly in the telecom $\mathrm{C}$ band [61], which relies on the continuous development of material growth technology. It thus seems feasible that our proposal provides a promising experimental route toward high-rate quantum key distribution with potential for proof-of-principle experiments with current technology.

\section{ACKNOWLEDGMENTS}

We would like to thank Dirk Englund, Matthias Christandl, Martin Hayhurst Appel, Ralf Riedinger, and Mihir Bhaskar for many valuable discussions. P. L. and A.S.S. gratefully acknowledge financial support from the Danish National Research Foundation (Center of Excellence "Hy-Q," Grant No. DNRF139), the European Research Council (ERC Advanced Grant "SCALE"), and the European Union's Horizon 2020 research and innovation programme under Grant Agreement No. 820445 and project name Quantum Internet Alliance. J. B. acknowledges financial support from VILLUM FONDEN via the QMATH Centre of Excellence (Grant No. 10059). T. S. was supported by the European Union's Horizon 2020 research and innovation program under the Marie Skłodowska Curie Grant Agreement No. 753067 (OPHOCS) and the Federal Ministry of Education and Research of Germany (BMBF, Project No. DiNOQuant13N14921). Work at Harvard was 
supported by NSF, CUA, NSF EFRI, ARL, and Vannevar Bush Fellowship.

[1] T. D. Ladd, F. Jelezko, R. Laflamme, Y. Nakamura, C. Monroe, and J. L. O'Brien, Quantum Computers, Nature (London) 464, 45 (2010).

[2] N. Gisin, G. Ribordy, W. Tittel, and H. Zbinden, Quantum Cryptography, Rev. Mod. Phys. 74, 145 (2002).

[3] H. J. Kimble, The Quantum Internet, Nature (London) 453 , 1023 (2008).

[4] S. Wehner, D. Elkouss, and R. Hanson, Quantum Internet: A Vision for the Road Ahead, Science 362, eaam9288 (2018).

[5] H.-J. Briegel, W. Dür, J. I. Cirac, and P. Zoller, Quantum Rrepeaters: The Role of Imperfect Local Operations in Quantum Communication, Phys. Rev. Lett. 81, 5932 (1998).

[6] W. J. Munro, K. Azuma, K. Tamaki, and K. Nemoto, Inside Quantum Repeaters, IEEE J. Sel. Top. Quantum Electron. 21, 78 (2015).

[7] S. Muralidharan, L. Li, J. Kim, N. Lütkenhaus, M. D. Lukin, and L. Jiang, Optimal Architectures for Long Distance Quantum Communication, Sci. Rep. 6, 20463 (2016).

[8] A. Seri, A. Lenhard, D. Rieländer, M. Gündoğan, P. M. Ledingham, M. Mazzera, and H. de Riedmatten, Quantum Correlations between Single Telecom Photons and a Multimode On-Demand Solid-State Quantum Memory, Phys. Rev. X 7, 021028 (2017).

[9] J. Borregaard, A. S. Sørensen, and P. Lodahl, Quantum Networks with Deterministic Spin-Photon Interfaces, Adv. Quantum Technol. 2, 1800091 (2019).

[10] A. G. Fowler, D. S. Wang, C. D. Hill, T. D. Ladd, R. Van Meter, and L.C. L. Hollenberg, Surface Code Quantum Communication, Phys. Rev. Lett. 104, 180503 (2010).

[11] W. J. Munro, A. M. Stephens, S. J. Devitt, K. A. Harrison, and K. Nemoto, Quantum Communication without the Necessity of Quantum Memories, Nat. Photonics 6, 777 (2012).

[12] S. Muralidharan, J. Kim, N. Lütkenhaus, M. D. Lukin, and L. Jiang, Ultrafast and Fault-Tolerant Quantum Communication across Long Distances, Phys. Rev. Lett. 112, 250501 (2014).

[13] A. N. Glaudell, E. Waks, and J. M. Taylor, Serialized Quantum Error Correction Protocol for High-Bandwidth Quantum Repeaters, New J. Phys. 18, 093008 (2016).

[14] K. Azuma, K. Tamaki, and H.-K. Lo, All-Photonic Quantum Repeaters, Nat. Commun. 6, 6787 (2015).

[15] F. Ewert, M. Bergmann, and P. van Loock, Ultrafast LongDistance Quantum Communication with Static Linear Optics, Phys. Rev. Lett. 117, 210501 (2016).

[16] S.-W. Lee, T. C. Ralph, and H. Jeong, A Fundamental Building Block for All-Optical Scalable Quantum Networks, Phys. Rev. A 100, 052303 (2019).

[17] V. Scarani, H. Bechmann-Pasquinucci, N. J. Cerf, M. Dušek, N. Lütkenhaus, and M. Peev, The Security of Practical Quantum Key Distribution, Rev. Mod. Phys. 81, 1301 (2009).
[18] F. Ewert and P. van Loock, Ultrafast Fault-Tolerant LongDistance Quantum Communication with Static Linear Optics, Phys. Rev. A 95, 012327 (2017).

[19] M. Varnava, D. E. Browne, and T. Rudolph, Loss Tolerance in One-Way Quantum Computation via Counterfactual Error Correction, Phys. Rev. Lett. 97, 120501 (2006).

[20] M. Pant, H. Krovi, D. Englund, and S. Guha, Rate-Distance Tradeoff and Resource Costs for All-Optical Quantum Repeaters, Phys. Rev. A 95, 012304 (2017).

[21] D. Buterakos, E. Barnes, and S. E. Economou, Deterministic Generation of All-Photonic Quantum Repeaters from Solid-State Emitters, Phys. Rev. X 7, 041023 (2017).

[22] A. Reiserer, N. Kalb, G. Rempe, and S. Ritter, A Quantum Gate between a Flying Optical Photon and a Single Trapped Atom, Nature (London) 508, 237 (2014).

[23] T. G. Tiecke, J. D. Thompson, N. P. de Leon, L. R. Liu, V. Vuletić, and M. D. Lukin, Nanophotonic Quantum Phase Switch with a Single Atom, Nature (London) 508, 241 (2014).

[24] N. Kalb, A. Reiserer, S. Ritter, and G. Rempe, Heralded Storage of a Photonic Quantum Bit in a Single Atom, Phys. Rev. Lett. 114, 220501 (2015).

[25] S. Sun, H. Kim, Z. Luo, G. S. Solomon, and E. Waks, A Single-Photon Switch and Transistor Enabled by a SolidState Quantum Memory, Science 361, 57 (2018).

[26] See Supplemental Material at http://link.aps.org/ supplemental/10.1103/PhysRevX.10.021071 for details on spin-photon $\mathrm{CZ}$ gate, numerical optimization, sketch of experimental architecture, and comparison with other repeater schemes and direct transmission.

[27] H. Pichler, S. Choi, P. Zoller, and M. D. Lukin, Universal Photonic Quantum Computation via Time-Delayed Feedback, Proc. Natl. Acad. Sci. U.S.A. 114, 11362 (2017).

[28] G. T. Reed, G. Mashanovich, F. Y. Gardes, and D. J. Thomson, Silicon Optical Modulators, Nat. Photonics 4, 518 (2010).

[29] C. Sun et al., Single-Chip Microprocessor That Communicates Directly Using Light, Nature (London) 528, 534 (2015).

[30] Y. Ogiso, J. Ozaki, Y. Ueda, N. Kashio, N. Kikuchi, E. Yamada, H. Tanobe, S. Kanazawa, H. Yamazaki, Y. Ohiso, T. Fujii, and M. Kohtoku, Over $67 \mathrm{GHz}$ Bandwidth and $1.5 V V \pi$ InP-Based Optical IQ Modulator with n-i-p-n Heterostructure, J. Lightwave Technol. 35, 1450 (2017).

[31] S. Koeber, R. Palmer, M. Lauermann, W. Heni, D. L. Elder, D. Korn, M. Woessner, L. Alloatti, S. Koenig, P. C. Schindler, H. Yu, W. Bogaerts, L. R. Dalton, W. Freude, J. Leuthold, and C. Koos, Femtojoule Electro-optic Modulation Using a Silicon-Organic Hybrid Device, Light Sci. Appl. 4, e255 (2015).

[32] I. Krasnokutska, J.-L. J. Tambasco, X. Li, and A. Peruzzo, Ultra-Low Loss Photonic Circuits in Lithium Niobate on Insulator, Opt. Express 26, 897 (2018).

[33] C. Wang, M. Zhang, X. Chen, M. Bertrand, A. ShamsAnsari, S. Chandrasekhar, P. Winzer, and M. Lončar, Integrated Lithium Niobate Electro-optic Modulators Operating at CMOS-Compatible Voltages, Nature (London) 562, 101 (2018). 
[34] T. G. Tiecke, K. P. Nayak, J. D. Thompson, T. Peyronel, N. P. de Leon, V. Vuletić, and M. D. Lukin, Efficient FiberOptical Interface for Nanophotonic Devices, Optica 2, 70 (2015).

[35] R. N. Patel, T. Schröder, N. Wan, L. Li, S. L. Mouradian, E. H. Chen, and D. R. Englund, Efficient Photon Coupling from a Diamond Nitrogen Vacancy Center by Integration with Silica Fiber, Light Sci. Appl. 5, e16032 (2016).

[36] R. S. Daveau, K. C. Balram, T. Pregnolato, J. Liu, E. H. Lee, J. D. Song, V. Verma, R. Mirin, S. W. Nam, L. Midolo, S. Stobbe, K. Srinivasan, and P. Lodahl, Efficient FiberCoupled Single-Photon Source Based on Quantum Dots in a Photonic-Crystal Waveguide, Optica 4, 178 (2017).

[37] M. Arcari, I. Söllner, A. Javadi, S. L. Hansen, S. Mahmoodian, J. Liu, H. Thyrrestrup, E. H. Lee, J. D. Song, S. Stobbe, and P. Lodahl, Near-Unity Coupling Efficiency of a Quantum Emitter to a Photonic Crystal Waveguide, Phys. Rev. Lett. 113, 093603 (2014).

[38] F. Liu, A. J. Brash, J. O’Hara, L. M. P. P. Martins, C. L. Phillips, R. J. Coles, B. Royall, E. Clarke, C. Bentham, N. Prtljaga, I. E. Itskevich, L. R. Wilson, M. S. Skolnick, and A. M. Fox, High Purcell Factor Generation of Indistinguishable On-Chip Single Photons, Nat. Nanotechnol. 13, 835 (2018).

[39] J. L. Zhang, S. Sun, M. J. Burek, C. Dory, Y.-K. Tzeng, K. A. Fischer, Y. Kelaita, K. G. Lagoudakis, M. Radulaski, Z.-X. Shen, N. A. Melosh, S. Chu, M. Lončar, and J. Vučković, Strongly Cavity-Enhanced Spontaneous Emission from Silicon-Vacancy Centers in Diamond, Nano Lett. 18, 1360 (2018).

[40] D. Kim, S. G. Carter, A. Greilich, A. S. Bracker, and D. Gammon, Ultrafast Optical Control of Entanglement between Two Quantum-Dot Spins, Nat. Phys. 7, 223 (2011).

[41] R. E. Evans, M. K. Bhaskar, D. D. Sukachev, C. T. Nguyen, A. Sipahigil, M. J. Burek, B. Machielse, G. H. Zhang, A. S. Zibrov, E. Bielejec, H. Park, M. Lončar, and M. D. Lukin, Photon-Mediated Interactions between Quantum Emitters in a Diamond Nanocavity, Science 362, 662 (2018).

[42] N. Kalb, A. A. Reiserer, P. C. Humphreys, J. J. W. Bakermans, S. J. Kamerling, N. H. Nickerson, S. C. Benjamin, D. J. Twitchen, M. Markham, and R. Hanson, Entanglement Distillation between Solid-State Quantum Network Nodes, Science 356, 928 (2017).

[43] S. Mahmoodian, P. Lodahl, and A. S. Sørensen, Quantum Networks with Chiral-Light-Matter Interaction in Waveguides, Phys. Rev. Lett. 117, 240501 (2016).

[44] P. C. Maurer, G. Kucsko, C. Latta, L. Jiang, N. Y. Yao, S. D. Bennett, F. Pastawski, D. Hunger, N. Chisholm, M. Markham, D. J. Twitchen, J. I. Cirac, and M. D. Lukin, Room-Temperature Quantum Bit Memory Exceeding One Second, Science 336, 1283 (2012).

[45] D. D. Sukachev, A. Sipahigil, C. T. Nguyen, M. K. Bhaskar, R. E. Evans, F. Jelezko, and M. D. Lukin, Silicon-Vacancy Spin Qubit in Diamond: A Quantum Memory Exceeding 10 ms with Single-Shot State Readout, Phys. Rev. Lett. 119, 223602 (2017).

[46] H. Bluhm, S. Foletti, I. Neder, M. Rudner, D. Mahalu, V. Umansky, and A. Yacoby, Dephasing Time of GaAs
Electron-Spin Qubits Coupled to a Nuclear Bath Exceeding $200 \mu$ s, Nat. Phys. 7, 109 (2011).

[47] D. A. Gangloff, G. Éthier-Majcher, C. Lang, E. V. Denning, J. H. Bodey, D. M. Jackson, E. Clarke, M. Hugues, C. Le Gall, and M. Atatüre, Quantum Interface of an Electron and a Nuclear Ensemble, Science 364, 62 (2019).

[48] L.-M. Duan and H. J. Kimble, Efficient Engineering of Multiatom Entanglement through Single-Photon Detections, Phys. Rev. Lett. 90, 253601 (2003).

[49] A. Sipahigil, R. E. Evans, D. D. Sukachev, M. J. Burek, J. Borregaard, M. K. Bhaskar, C. T. Nguyen, J. L. Pacheco, H. A. Atikian, C. Meuwly, R. M. Camacho, F. Jelezko, E. Bielejec, H. Park, M. Lončar, and M. D. Lukin, An Integrated Diamond Nanophotonics Platform for Quantum Optical Networks, Science 354, 847 (2016).

[50] A. Delteil, Z. Sun, W.-b. Gao, E. Togan, S. Faelt, and A. Imamoğlu, Generation of Heralded Entanglement between Distant Hole Spins, Nat. Phys. 12, 218 (2016).

[51] L. . Santis, C. Antón, B. Reznychenko, N. Somaschi, G. Coppola, J. Senellart, C. Gómez, A. Lemaître, I. Sagnes, A. G. White, L. Lanco, A. Auffèves, and P. Senellart, A Solid-State Single-Photon Filter, Nat. Nanotechnol. 12, 663 (2017).

[52] A. Javadi, D. Ding, M. H. Appel, S. Mahmoodian, M. C. Löbl, I. Söllner, R. Schott, C. Papon, T. Pregnolato, S. Stobbe, L. Midolo, T. Schröder, A. D. Wieck, A. Ludwig, R. J. Warburton, and P. Lodahl, Spin-Photon Interface and Spin-Controlled Photon Switching in a Nanobeam Waveguide, Nat. Nanotechnol. 13, 398 (2018).

[53] M. Bayer, P. Hawrylak, K. Hinzer, S. Fafard, M. Korkusinski, Z. R. Wasilewski, O. Stern, and A. Forchel, Coupling and Entangling of Quantum States in Quantum Dot Molecules, Science 291, 451 (2001).

[54] S.-C. Tian, R.-G. Wan, C.-Z. Tong, Y.-Q. Ning, L. Qin, and Y. Liu, Giant Kerr Nonlinearity Induced by Tunneling in Triple Quantum Dot Molecules, J. Opt. Soc. Am. B 31, 1436 (2014).

[55] M. K. Bhaskar, R. Riedinger, B. Machielse, D. S. Levonian, C. T. Nguyen, E. N. Knall, H. Park, D. Englund, M. Lončar, D. D. Sukachev, and M. D. Lukin, Experimental Demonstration of Memory-Enhanced Quantum Communication, Nature (London) 580, 60 (2020).

[56] D. Najer, I. Söllner, P. Sekatski, V. Dolique, M. C. Löbl, D. Riedel, R. Schott, S. Starosielec, S. R. Valentin, A. D. Wieck, N. Sangouard, A. Ludwig, and R. J. Warburton, A Gated Quantum Dot Strongly Coupled to an Optical Microcavity, Nature (London) 575, 622 (2019).

[57] B. Kambs, J. Kettler, M. Bock, J. N. Becker, C. Arend, A. Lenhard, S. L. Portalupi, M. Jetter, P. Michler, and C. Becher, Low-Noise Quantum Frequency Down-Conversion of Indistinguishable Photons, Opt. Express 24, 22250 (2016).

[58] A. Dréau, A. Tchebotareva, A. El Mahdaoui, C. Bonato, and R. Hanson, Quantum Frequency Conversion of Single Photons from a Nitrogen-Vacancy Center in Diamond to Telecommunication Wavelengths, Phys. Rev. Applied 9, 064031 (2018).

[59] M. Bock, P. Eich, S. Kucera, M. Kreis, A. Lenhard, C. Becher, and J. Eschner, High-Fidelity Entanglement be- 
tween a Trapped Ion and a Telecom Photon via Quantum Frequency Conversion, Nat. Commun. 9, 1998 (2018).

[60] F. Vedovato, C. Agnesi, M. Tomasin, M. Avesani, J.-Å. Larsson, G. Vallone, and P. Villoresi, Postselection-Loophole-Free Bell Violation with Genuine Time-Bin Entanglement, Phys. Rev. Lett. 121, 190401 (2018).
[61] C. Nawrath, F. Olbrich, M. Paul, S. L. Portalupi, M. Jetter, and P. Michler, Coherence and Indistinguishability of Highly Pure Single Photons from Non-Resonantly and Resonantly Excited Telecom C-Band Quantum Dots, Appl. Phys. Lett. 115, 023103 (2019). 